\title{
Endoport-assisted Neuroendoscopic Techniques Used in the Resection of Intraventricular Lesions
}

\author{
Sungel $\mathrm{XIE}^{1^{*}}$, Ling $\mathrm{XU}^{1 *}$, Ke WANG ${ }^{2}$, Fei-Ji SUN ${ }^{1}$, Ming-Xiang $\mathrm{XIE}^{1,2}$, Pei WANG ${ }^{1}$, Shun-Wu XIAO ${ }^{1,2}$ \\ ${ }^{1}$ The Affiliated Hospital of Zunyi Medical University, Department of Neurosurgery, Guizhou, China \\ 2Zunyi Medical University, Guizhou, China \\ ${ }^{*}$ These authors contributed equally to this article.
}

Corresponding author: Shun-Wu XIAO xswlove1976@126.com

\section{ABSTRACT}

AIM: To investigate the safety and efficacy of endoport-assisted endoscopic techniques when used to remove intraventricular lesions.

MATERIAL and METHODS: Data of patients with intraventricular lesions who were surgically treated by endoport-assisted endoscopic resection between January 2018 and February 2019 were retrospectively reviewed. The surgical procedures, complications and outcomes were analyzed.

RESULTS: A total of 11 patients, with a mean age of 33 years (5-70 years) were included in the study. The mean Karnofsky Performance Scale (KPS) score evaluated on admission was $50.0 \pm 7.0$. Lesions located in the unilateral ventricle, the third ventricle and multiple sites of ventricles were recorded in 7, 2 and 2 patients, respectively. The average lesion size was $3.4 \pm 0.4 \mathrm{~cm}(2-6$ $\mathrm{cm})$. Gross-total removal of all lesions was achieved, and all patients experienced a stable recovery after operations except for one hemorrhage and one visual field defect occurring in two patients in the early postoperative period. With a follow-up of 6-19 months, dysfunctions and complications occurring pre- or postoperation gradually recovered to different degrees. The mean KPS score was $85.5 \pm 4.3$ at the last follow-up, and no tumor recurrence was observed in any of the patients.

CONCLUSION: Endoport-assisted endoscopic techniques could be a simple, minimally invasive surgical method in the resection of lesions located in the lateral ventricle, the third ventricle, or both with acceptable surgical complications occurring in patients.

KEYWORDS: Endoport-assisted, Neuroendoscopy, Intraventricular, Surgery

ABBREVIATIONS: KPS: Karnofsky performance scale, MRI: Magnetic resonance images, CSF: Cerebrospinal fluid

\section{INTRODUCTION}

$\tau$ ntraventricular lesions are deep-seated, and they cannot be easily approached without injuring nerve tissue. One of the important surgical principles of ventricular surgery is to remove the lesion to the greatest extent and avoid extra neurological injury (30). Neuroendoscopy has always been viewed as an important tool for the treatment of ventricular diseases. With the development of endoscopic techniques, endoscopes are used not only to assist in biopsy but have also evolved into a tool used throughout the entire process of resection of intraventricular lesions $(2,5,13,19,27)$.

However, resection of intraventricular lesions requires creating a special passage through the cerebral cortex or the corpus callosum, which typically requires the use of brain retractors. Traditional brain spatulas are prone to injuring the cortex or nerve tracts when pulling the cortical and subcortical structures.
Sungel XIE (D) : 0000-0002-5100-2773

Ling XU (D) : 0000-0001-7077-3613

Ke WANG (D) : 0000-0002-5100-2773
Fei-Ji SUN (D) : 0000-0002-7889-5575

Ming-Xiang XIE (D) : 0000-0001-9094-5427

Pei WANG (D) : 0000-0002-6858-1038
Shun-Wu XIAO (D) : 0000-0002-3690-4963 
Instead, a circular retractor, including endoscope channels, such as endoport, can equally disperse the pulling pressure to reduce such injuries. Endoport-assisted endoscopic surgery combines both advantages of the endoscope and the circular retractor, thereby facilitating safe and effective removal of intraventricular lesions $(20,27,29)$. However, the surgical strategy and outcomes of this technique are still insufficiently reported; hence, we retrospectively reviewed our surgical cases to evaluate the safety and efficacy of endoport-assisted endoscopic techniques when used to remove intraventricular lesions by transcortical approaches.

\section{MATERIAL and METHODS}

\section{Patient Population}

Between January 2018 and February 2019, 11 patients were diagnosed with intraventricular lesions and were surgically treated by endoport-assisted endoscopic resection in our institute. Data on patient demographics, lesion characteristics, surgical procedures and complications with respect to endoport application were retrospectively reviewed. The Research Ethics Committee of our institute approved the study.

All neuroimaging data were reviewed by 2 independent neuroradiologists. The lesion size was evaluated as the lesion equivalent diameter $(\mathrm{abc})^{1 / 3}$, where $\mathrm{a}, \mathrm{b}$, and $\mathrm{c}$ represent 3 diameter measurements obtained on axial, sagittal and coronal T1-weighted magnetic resonance images (MRI), respectively. Complete resection was determined by postoperative MRI. The Karnofsky Performance Scale (KPS) scoring was used to evaluate the patient's neurological status. Follow-up was obtained in the clinic and/or by telephone and ended in August 2019.

\section{Surgical Procedure}

The endoport-assisted endoscopic transcortical approach was used to resect intraventricular lesions (Figure $1 \mathrm{~A}-\mathrm{H}$ ). Different surgical entry points on the cerebral cortex were planned according to the specific location of the lesion in the cerebral ventricles, and the bone window of operations was typically as large as $3.0 \mathrm{~cm} \times 2.5 \mathrm{~cm}$. 1) The trans-middle frontal cortical approach was used for lesions in the frontal compartment or the foramen of Monro of lateral ventricles; 2) The trans-superior parietal cortical approach was selected for lesions at the body of lateral ventricles; 3) The trans-occipital cortical approach was planned for lesions at the trigone of lateral ventricles; 4) Lesions at the center and back of the third ventricle except for growing from the pineal region could be removed through the trans-middle frontal cortical, choroidal fissure and expanded foramen approach.

Various endoport types (TC211507, or TC171107, Vycor Medical, Inc., Boca Raton, FL), including 3 lengths (3, 5 and 7 $\mathrm{cm}$ ) and 4 diameters $(12,17,21$ and $28 \mathrm{~mm})$, could be chosen depending on the lesion size and location (27). Accordingly, if the neoplasm is large in size and deep, a larger endoport should be used, such as the $7 \mathrm{~cm} \times 21 \mathrm{~mm}$ endoport used in our institute, and the bone window could be expanded.
For lesions located in the third ventricle with obstructive hydrocephalus, a fistula could be made at the bottom of the third ventricle after resection. Navigation was used for preoperative planning and incision design and validation.

A puncture needle was inserted into the cerebral ventricle to orient lesions under navigation guidance. Then, the endoport was implanted along the puncture needle cannula, and the endoscope was inserted into the endoport. Access to the cerebral ventricle was then visualized directly with an endoscope (4-mm diameter, 18-cm length, 0-degree lenses; Karl Storz GmbH \& Co., Tuttlingen, Germany), and the lesion could be exposed (Figure 2A-I). The endoscope was fixed on the air-driven arm, and both hands were free for surgical manipulations.

Lesions that were smaller than $1.5 \mathrm{~cm}$ and hard in texture could be completely removed en bloc after disrupting the blood supply and dissecting their boundaries. If lesions were greater than $1.5 \mathrm{~cm}$, intracapsular decompression was first performed for the soft tissue, and piecemeal resection was performed for the hard tissue. For colloid cysts at ventricles, a small incision must be made first at the cyst wall, and the fluid in the cyst should be slowly removed using a suction unit before the cyst wall and blood vessels are completely separated to avoid the cyst fluid entering the cerebral ventricles and resulting in aseptic ventriculitis (Figure 2A-I).

After resection, fewer hemostatic materials should be placed at the surgical site, and slight bipolar hemostasis or Surgicel compression could be performed if there are petechiae. Warm physiological saline was used to perfuse ventricles to expel air. Then, a drainage tube was inserted. The endoport was withdrawn under direct endoscopic visualization. Then, the dura was sutured, and the bone flap was replaced. The drainage tube could be removed on the second day if there was no ventricular bleeding after surgery.

\section{RESULTS}

\section{Patients and Surgical Outcomes}

With a total of 11 patients ( 8 female and 3 male) in the study, the mean age was $33.1 \pm 7.5$ years ( $5-70$ years), and the mean KPS score was $50.0 \pm 7.0$ on admission. Seven patients suffered preoperative hydrocephalus, and all patients complained of at least one symptom, including headache (10), vomiting (2), memory decline (4), disturbance of consciousness (3), urinary incontinence (2) and convulsion (1) (Table I). In particular, two patients (No. 1 and No. 8, Table I) who primarily complained about headache suffered progressing symptoms and subsequently experienced unconsciousness after admission with concurrent convulsion in case 1 and urinary incontinence in case 8 . There were 7 cases with lesions located exclusively in lateral ventricles and 2 cases located exclusively in the third ventricle. In addition, 2 cases were found with lesions located in multiple ventricles. The average lesion size was $3.4 \pm 0.4$ $\mathrm{cm}(2-6 \mathrm{~cm})$.

Gross-total removal was achieved for all lesions. The histological pathology results are shown in Table I. After 

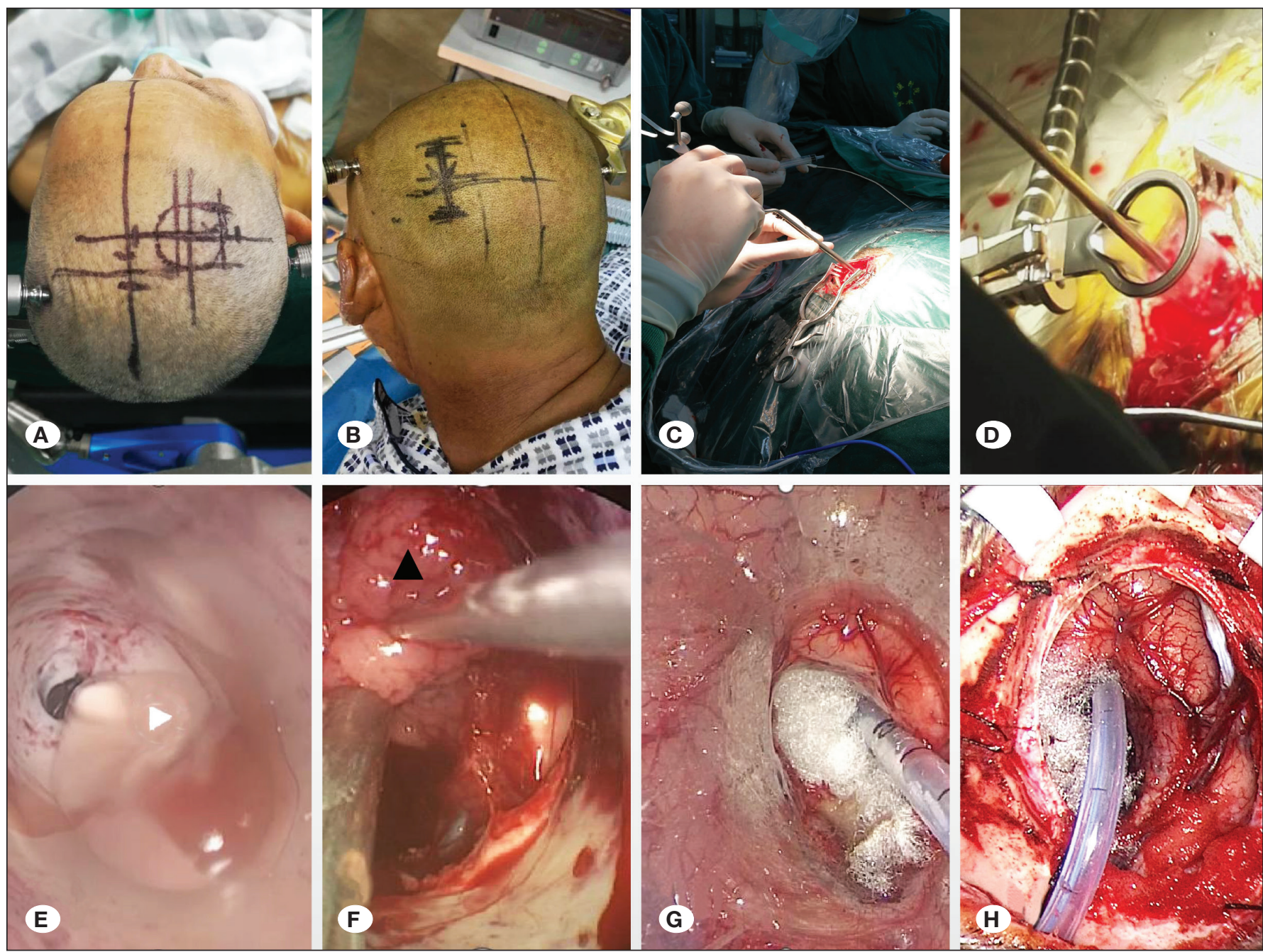

Figure 1: Pictures (A-H) depicting the main procedures of endoport-assisted endoscopic resection of intraventricular tumors. Pictures (A, B) displaying two patients' positions in the supine position (A) for the trans-middle frontal cortical approach and prone position (B) for the trans-superior parietal cortical approach. Pictures (C, D) showing the intraoperative navigation towards the tumor (C) and endoport implantation (D). Pictures (E-H) illustrating the cerebrospinal fluid (white triangle) flowing from the ventricle (E), tumor (black triangle) being exposed and resected $(\mathbf{F})$, and hemostasis upon endoport extraction (G) and implantation of drainage tube $(\mathbf{H})$ after tumor removal.

surgery, all patients achieved a stable recovery. Complications in the early period were found in 2 patients, including hematoma (1) and visual field defects (1). Close observation was administered to the patient who had postoperative bleeding due to his slight symptoms and an unchanged hematoma volume. The average KPS score evaluated at discharge was $73.6 \pm 4.3$.

During 6-19 months of follow-up, all patients showed an obvious improvement in neurological dysfunction with an average KPS score recorded at $85.5 \pm 4.3$ at the most recent evaluation. In particular, the postoperative complications of visual defects in a patient completely improved by the second month after the operation. Follow-up was continued, and no recurrence was noted in any of the patients.

\section{DISCUSSION}

The use of endoport as a neurosurgical tubular working cannula and a retractor can be traced to 1988 when Kelly et al. reported using stereotactic tubular retractors to resect deep lesions in the brain and hippocampus and amygdala since 1984 (18). Subsequently, many researchers reported similar surgeries in which tubular cannulas were used to resect intracranial lesions under stereotactic systems $(3,22,26)$, including the combined use of endoscopy. With the development of frameless navigation techniques, tubular cannulas have been increasingly used. In 2000, Nishihara et al. first reported the use of a transparent plastic sheath $(6 \mathrm{~mm}$ in diameter) with endoscopy to eliminate intracranial hematomas (21). In 2002, Jho and Alfieri reported the use of an expandable ethylene plastic with endoscopy to resect tumors in the third ventricle (15). Subsequently, an increasing number of researchers 

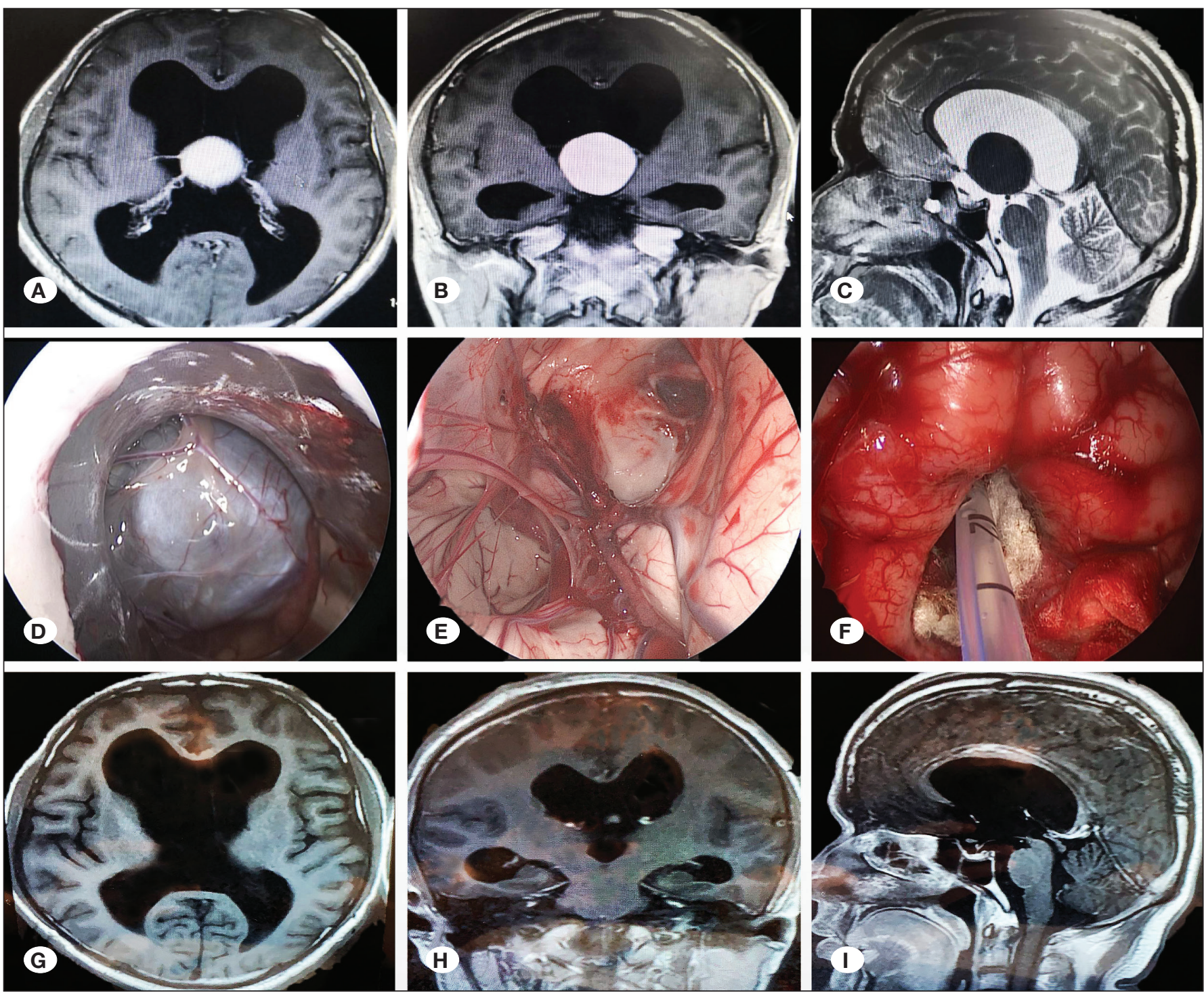

Figure 2: This 31-year-old man experienced severe headache and vomiting on admission. Preoperative imaging (A-C) of T1-weighted axial (A) and coronal (B) and T2-weighted sagittal (C) MR images on admission showed a large colloid cyst involving the third ventricle with obvious obstructive hydrocephalus. An endoport-assisted endoscopic trans-middle frontal cortical, choroidal fissure and expanded foramen approach was planned to remove the neoplasm, and pictures (D-F) showing the exposure of cyst (D), total removal (E) and implantation of drainage tube (F). Postoperative MR imaging (G-I) of T1-weighted Gd-enhanced axial (G), coronal (H) and sagittal (I) images at the seventh month after surgery illustrated gross total resection of the lesion.

have reported the resection of intracranial lesions under an endoscopic working cannula $(8,11,14,17)$, including resection of lesions at problematic sites, such as the brainstem (16). With a wide review of previous literature, we found that the tubular working cannula could be used to excise a variety of lesions, including intracranial primary or metastatic tumors, cysts, and cerebrovascular diseases. The involved sites include the cerebral lobes, cerebral ventricles, basal ganglia, brainstem, cerebellum, cerebellopontine angle, and spinal cord $(6,9,10,12,16,23-25,28)$.

\section{The Advantages of Endoport}

The following superiorities of using the surgical tubular working cannula supplied by endoport were noted: 1) only a small skin incision and a small bone window are needed; 2) compared with conventional retractors, the circular or oval working cannula and blunt core of endoport uniformly distribute traction pressure in a conical shape, which reduces the traction injury of brain tissues; 3) creating a bloodless working cannula environment can facilitate surgical manipulations; 4) iatrogenic injury caused by equipment can be reduced when the physician operates within the working cannula, and this also reduces the thermal injury caused by the use of endoscopy; and 5) adjusting the direction of the working cannula can expose a large area of the lesion and does not significantly increase brain tissue injury. 


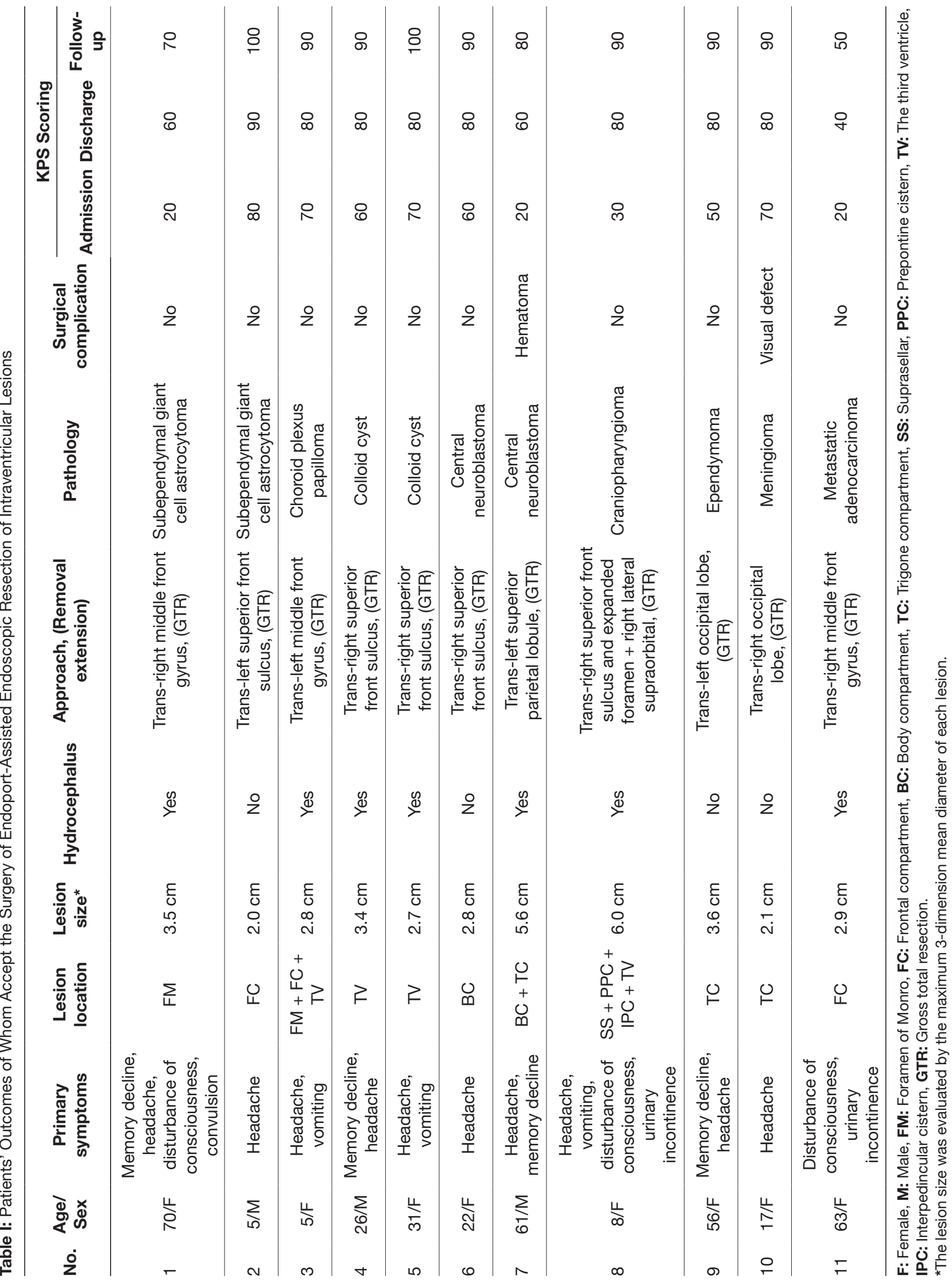


Lesions can be removed at the tubular cannula under the microscope as well, and some researchers have compared the merits and disadvantages of microscopic and endoscopic surgeries $(1,4,6,12)$. Some researchers thought that microscopic methods are more convenient and could provide surgeons direct and high-definition surgical fields and better control intraoperative bleeding (12). We found that it was indeed convenient to use the microscope, and that endoscope had difficulties in attaining hemostasis for rapid or massive bleeding events especially for surgeons with minimal experience. However, endoport-assisted microsurgery requires improved lighting for the resection of brain lesions in deep sites or with large sizes, and endoscopic methods exhibit the strength of close observation, which could compensate for that defect. With the development of endoscopic techniques, it is possible to use endoscopy combined with microscopy for the entire neurosurgical process. In particular, endoscopy is more suitable for large and/or deep-located lesions, such as intraventricular neoplasms.

\section{Key Points for Endoport Surgery}

There are several key points for endoport surgery: 1) the position of the endoscope and endoport should be placed in relation to the location of the lesion. In general, the endoscope is fixed in front of the cannula wall to reduce restrictions on the surgical equipment. 2) Different endoport sizes were selected according to the lesion site, size, texture and blood supply and even patients' head size. Tumors at the anterior part of the lateral ventricle are typically small when discovered, and a small endoport with a length of $7 \mathrm{~cm}$ and a diameter of $17 \mathrm{~mm}$ or $21 \mathrm{~mm}$ could be used in an adult. In contrast, meningiomas at the trigone of the lateral ventricle are typically large when discovered and generally exhibit a hard texture or rich blood supply; thus, a large endoport $7 \mathrm{~cm}$ in length and $28 \mathrm{~mm}$ in diameter could be used. 3) The cerebral sulci and fissure surface and deep regions typically contain veins and arteries, and there is a risk of vascular injury when an endoport is inserted. Thus, more attention should be paid when operations are performed. In the present study, we used a transsulcal approach for 6 patients and a transgyrus approach for 5 patients, and no cannula bleeding occurred. 4) We did not perform routine cortical resection to approach the cerebral ventricle, but we dissected the cortex to confirm access into the cerebral ventricle through a puncture needle. 5) Operations in ventricles commonly lead to loss of CSF and collapse of brain tissue. The shoulders and head are typically lifted in a high position to reduce CSF loss. Before the end of surgery, warm physiological saline was used to perfuse the surgical cavity to expel air, and then a drainage tube was inserted. 6) For large tumors with abundant blood supply, the insertion of drainage tubes is conventionally recommended. For patients with small tumors, where hemostasis can be controlled, a drainage tube is not used to reduce the incidence of infection and other complications.

\section{Lowering Surgical Complications}

Shapiro et al. reviewed the current literature of English articles that reported tubular retractors of the ViewSite Brain Access System (VBAS; Vycor Medical Inc.) to assess its surgical ben- efits and complications. They found a total of 12 publications ( $n=106$ patients) between 2005 and 2019 that reported the use of VBAS retractors for tumor resections, hematoma evacuations, cyst removal, foreign body extractions, and multiple sclerosis. In different brain areas, only 3 short-term postoperative complications were linked to the retractor with an overall complication rate of $2.8 \%(27)$.

Eichberg et al. also performed a retrospective analysis of transtubular colloid cyst resections in their 10 cases and 77 cases in the literature to evaluate the efficacy and safety profile of transtubular techniques. These researchers found that the overall incidence of complications was $7.8-10 \%$, and no postoperative seizures or venous injuries were recorded in their cases (7). In our study, complications, including bleeding and new neurological injury of the visual field, were observed in one patient each. The cause of bleeding was the absence of gradual examination for apparent bleeding when withdrawing the endoport, and Surgicel or Surgiflo was not gradually placed at the tunnel. Therefore, bleeding was completely avoided in subsequent cases. Visual field defects might be attributed to the injury of the optic radiation when the endoport passed through the occipital cortex to the lesion in the trigone of the lateral ventricles. However, this patient experienced a recovery of visual fields after one month of follow-up, and use of the medial trans-precuneus approach was recommend to avoid such injury (31).

\section{Limitations}

The sample size of this study was relatively small, and inherent bias existed in this retrospective study with a short followup duration. In addition, the management of intraventricular lesions also involves advanced functional assessment of conduction bundles. In the future, we need to strengthen the research in these areas and further assess whether endoport can reduce or prevent injury to the conduction bundles, and we should evaluate the effects of endoport on the duration of surgery, blood loss, and treatment results compared with conventional methods.

\section{CONCLUSIONS}

Endoport-assisted endoscopic techniques could represent a simple, minimally invasive surgical method to remove lesions in the lateral ventricles, the third ventricle or both by a transcortical approach with acceptable postoperative complications occurring in patients, especially reducing traction injury of the brain. However, more surgical cases should be reported to support this conclusion.

\section{REFERENCES}

1. Alexopoulos G, Prim M, Khan M, Quadri N, Urquiaga JF, El Tecle N, Coppens J: Minimally invasive evacuation of severe intraventricular hemorrhage using the brainpath endoportassisted microsurgical system. World Neurosurg 134: e540-e548, 2020

2. Barber SM, Rangel-Castilla L, Baskin D: Neuroendoscopic resection of intraventricular tumors: A systematic outcomes analysis. Minim Invasive Surg 2013:898-753, 2013 
Xie S. et al: Neuroendoscopic Resection of Intraventricular Lesions

3. Barlas O, Karadereler S: Stereotactically guided microsurgical removal of colloid cysts. Acta Neurochir (Wien) 146:1199-204, 2004

4. Cohen-Gadol AA: Minitubular transcortical microsurgical approach for gross total resection of third ventricular colloid cysts: Technique and assessment. World Neurosurg 79:207. e7-10, 2013

5. Depreitere B, Dasi N, Rutka J, Dirks P, Drake J: Endoscopic biopsy for intraventricular tumors in children. J Neurosurg 106:340-346, 2007

6. Ding D, Starke RM, Crowley RW, Liu KC: Endoport-assisted microsurgical resection of cerebral cavernous malformations. J Clin Neurosci 22:1025-1029, 2015

7. Eichberg DG, Buttrick SS, Sharaf JM, Snelling BM, Shah AH, Ivan ME, Komotar RJ: Use of tubular retractor for resection of colloid cysts: Single surgeon experience and review of the literature. Oper Neurosurg (Hagerstown) 16:571-579, 2019

8. Engh JA, Lunsford LD, Amin DV, Ochalski PG, FernandezMiranda J, Prevedello DM, Kassam AB: Stereotactically guided endoscopic port surgery for intraventricular tumor and colloid cyst resection. Neurosurgery 67:ons198-204; discussion ons-5, 2010

9. Fahim DK, Relyea K, Nayar VV, Fox BD, Whitehead WE, Curry DJ, Luerssen TG, Jea A: Transtubular microendoscopic approach for resection of a choroidal arteriovenous malformation. J Neurosurg Pediatr 3:101-104, 2009

10. Greenfield JP, Cobb WS, Tsouris AJ, Schwartz TH: Stereotactic minimally invasive tubular retractor system for deep brain lesions. Neurosurgery 63:334-339; discussion 339-340, 2008

11. Harris AE, Hadjipanayis CG, Lunsford LD, Lunsford AK, Kassam AB: Microsurgical removal of intraventricular lesions using endoscopic visualization and stereotactic guidance. Neurosurgery 62 Suppl 2:622-629, 2008

12. Hong CS, Prevedello DM, Elder JB: Comparison of endoscope- versus microscope-assisted resection of deepseated intracranial lesions using a minimally invasive port retractor system. J Neurosurg 124:799-810, 2016

13. Ibanez-Botella G, Segura M, De Miguel L, Ros B, Arraez MA: Purely neuroendoscopic resection of intraventricular tumors with an endoscopic ultrasonic aspirator. Neurosurg Rev 42(4):973-982, 2019

14. Ichinose T, Goto T, Morisako H, Takami T, Ohata K: Microroll retractor for surgical resection of brainstem cavernomas. World Neurosurg 73:520-522, 2010

15. Jho HD, Alfieri A: Endoscopic removal of third ventricular tumors: A technical note. Minim Invasive Neurosurg 45:114119, 2002

16. Jo KW, Shin HJ, Nam DH, Lee JI, Park K, Kim JH, Kong DS: Efficacy of endoport-guided endoscopic resection for deepseated brain lesions. Neurosurg Rev 34:457-463, 2011

17. Kassam AB, Engh JA, Mintz AH, Prevedello DM: Completely endoscopic resection of intraparenchymal brain tumors. $J$ Neurosurg 110:116-123, 2009
18. Kelly PJ, Goerss SJ, Kall BA: The stereotaxic retractor in computer-assisted stereotaxic microsurgery. Technical note. J Neurosurg 69:301-306, 1988

19. Mohanty A, Thompson BJ, Patterson J: Initial experience with endoscopic side cutting aspiration system in pure neuroendoscopic excision of large intraventricular tumors. World Neurosurg 80:655.e15-21, 2013

20. Moosa S, Ding D, Mastorakos P, Sheehan JP, Liu KC, Starke RM: Endoport-assisted surgical evacuation of a deep-seated cerebral abscess. J Clin Neurosci 53:269-272, 2018

21. Nishihara T, Teraoka A, Morita A, Ueki K, Takai K, Kirino T: A transparent sheath for endoscopic surgery and its application in surgical evacuation of spontaneous intracerebral hematomas. Technical note. J Neurosurg 92:1053-1055, 2000

22. Otsuki T, Jokura H, Yoshimoto T: Stereotactic guiding tube for open-system endoscopy: A new approach for the stereotactic endoscopic resection of intra-axial brain tumors. Neurosurgery 27:326-330, 1990

23. Palmer S: Use of a tubular retractor system in microscopic lumbar discectomy: 1 year prospective results in 135 patients. Neurosurg focus 13:E5, 2002

24. Raza SM, Recinos PF, Avendano J, Adams H, Jallo GI, Quinones-Hinojosa A: Minimally invasive trans-portal resection of deep intracranial lesions. Minim Invasive Neurosurg 54: 5-11, 2011

25. Recinos PF, Raza SM, Jallo GI, Recinos VR: Use of a minimally invasive tubular retraction system for deep-seated tumors in pediatric patients. J Neurosurg Pediatr 7:516-521, 2011

26. Sabanci PA, Aras Y, Ali A, Unal TC, Dolen D, Sencer S, Izgi N, Unal F, Barlas O: Transcortical removal of third ventricular colloid cysts: Comparison of conventional, guided microsurgical and endoscopic approaches and review of the literature. Turk Neurosurg 27:546-557, 2017

27. Shapiro SZ, Sabacinski KA, Mansour SA, Echeverry NB, Shah SS, Stein AA, Snelling BM: Use of vycor tubular retractors in the management of deep brain lesions: A review of current studies. World Neurosurg 133:283-290, 2020

28. Shoakazemi A, Evins AI, Burrell JC, Stieg PE, Bernardo A: A $3 \mathrm{D}$ endoscopic transtubular transcallosal approach to the third ventricle. J Neurosurg 122:564-573, 2015

29. Sujijantarat N, Tecle NE, Pierson M, Urquiaga JF, Quadri NF, Ashour AM, Khan MQ, Buchanan P, Kumar A, Feen E, Coppens $\mathrm{J}$ : Trans-sulcal endoport-assisted evacuation of supratentorial intracerebral hemorrhage: Initial single-institution experience compared to matched medically managed patients and effect on 30-day mortality. Oper Neurosurg (Hagerstown) 14:524531,2018

30. Yasargil MG, Abdulrauf SI: Surgery of intraventricular tumors. Neurosurgery 62:1029-1040; discussion 1040-1041, 2008

31. Zhu W, Xie T, Zhang X, Ma B, Wang X, Gu Y, Ge J, Xu W, Hu F, Zhang Y, Li Q, Yu Y, Zhou H, Jiang Y, Li W: A solution to meningiomas at the trigone of the lateral ventricle using a contralateral transfalcine approach. World Neurosurg 80:167172,2013 